\title{
An efficient adaptive hierarchical sliding mode control strategy using neural networks for 3D overhead cranes
}

\author{
Viet-Anh LE ${ }^{1} \quad$ Hai Xuan LE ${ }^{1} \quad$ Linh NGUYEN ${ }^{2} \quad$ Minh Xuan PHAN ${ }^{1}$ \\ ${ }^{1}$ Department of Automatic Control, Hanoi University of Science and Technology, Hanoi, 10000, Vietnam \\ ${ }^{2}$ Centre for Autonomous Systems, University of Technology, Sydney, New South Wales 2007, Australia
}

\begin{abstract}
In this paper, a new adaptive hierarchical sliding mode control scheme for a 3D overhead crane system is proposed. A controller is first designed by the use of a hierarchical structure of two first-order sliding surfaces represented for two actuated and unactuated subsystems in the bridge crane. Parameters of the controller are then intelligently estimated, where uncertain parameters due to disturbances in the 3D overhead crane dynamic model are proposed to be represented by radial basis function networks whose weights are derived from a Lyapunov function. The proposed approach allows the crane system to be robust under uncertainty conditions in which some uncertain and unknown parameters are intractable to be determined. Moreover, stability of the sliding surfaces is proved to be guaranteed. Effectiveness of the proposed approach is then demonstrated by implementing the algorithm in both synthetic and real-life systems, where the results obtained by our method are highly promising.
\end{abstract}

Keywords: 3D overhead crane, adaptive hierarchical sliding mode control, neural network, radial basis function.

\section{Introduction}

An overhead crane, also known as a bridge crane, plays a critical role in many factories or industries to transport heavy payloads or hazardous wastes/materials from one place to another place or in harbour ports to load and unload cargo from ships ${ }^{[1],[2]}$. The overhead crane is classified into a class of under-actuated mechanical systems, where a number of outputs is greater than a number of control inputs. On the other hand, in modern factories, due to requirement of increasing productivity, a bridge crane system is frequently operated at high speed, which mainly leads to payload oscillations severely affecting on safety of operators, cargo or surrounding items ${ }^{[3]}$. Moreover, the cargo oscillations are nonlinear and highly coupled with motions of actuators such as bridge, trolley or hoist ${ }^{[4]}$. Due to these complicated dynamics and non-holonomic behaviours, an overhead crane is deemed to be difficult to control. Therefore, a fundamental problem in designing a controller for a $3 \mathrm{D}$ overhead crane system is that control law is required to minimize swings of a cargo while moving it fast to a desired destination $^{[5]}$.

In order to optimally address the aforesaid control issues for a crane system, many approaches have been proposed in the past decades. All those control laws can be primarily classified into two categories of the open-loop and closed-loop methods. The former is quite simple since it is straightforward to implement, no additional angle sensor requirement ${ }^{[6]-[8]}$. Nonetheless, the open-loop control technique is very sensitive to external disturbances such as wind when a $3 \mathrm{D}$ bridge crane is utilized outdoors ${ }^{[9]}$. In contrast to the open-loop approach, the closed/feedback-loop control method employs sensor measurements and system state estimations to adjust actuators to obtain desired outputs, which enables a crane system to be less sensitive to external disturbances as well as uncertainties ${ }^{[10]}$. For instance, in [11], by using LaSalle's invariance set theorem, Fang et al. proposed a proportional-derivative control scheme to adjust motions of the bridge and cargo in a two degree-of- freedom overhead crane. They then relied on both squared and kinetic energies to design two nonlinear control laws to enhance performance of the system in transient states. Likewise, the work in [12] developed a nonlinear two closedloop controller to not only fast drive a payload on a desired trajectory but also eliminate its oscillations. In the context of partial feedback linearization, the authors in [13] fully incorporated nonlinear dynamics of a $2 \mathrm{D}$ crane system into a controller that simultaneously controls positions of a trolley and hoisted payload and minimizes load sway angles. Moreover, Le et al. in their works [14], [15] enhanced the nonlinear partial feedback linearization controller for a $3 \mathrm{D}$ overhead crane system. By considering nonlinear feedback of actuated and un-actuated states in a superposition manner, a three input control law was designed to asymptotically stabilize the states of the crane system. In addition to the approaches based on the deterministic models of a crane system, a fuzzy logic controller, which is designed by representing an overhead crane by not a mathematical but fuzzy model, has been widely employed in the crane control community ${ }^{[16]-[18]}$. Due to its strong adaptability to complexity and nonlinearity of a crane, the fuzzy logic control law considerably benefits control actions of a 3D overhead crane.

Nonetheless, if one is interested in robustness of a crane system regardless its uncertainties and nonlinearities, the sliding mode control (SMC) ${ }^{[19]-[24]}$ is the best candidate when considering design of a controller. For an underactuated overhead crane, a SMC scheme is not only robust but also efficient to control the system even it is under uncertain conditions. Nevertheless, there still exists challenging issues regarding control in a crane system. For instance, how to define sliding surfaces so as to guarantee stability of actuated and un-actuated subsystems while the number of control inputs is smaller than that of actuators is still a fully unsolved question for control law makers. In literature, there have been some works proposed to address the issue. In [19],[21] a sliding surface is constituted by linearly combining a sliding surface of actuated states and errors of 
un-actuated states. Furthermore, the authors in [20], [25] first developed an intermediate variable based on state errors, which then allowed them to form a second-level sliding surface.

Among approaches of defining a sliding surface for a SMC scheme, an efficient technique named hierarchical SMC (HSMC) particularly interests researchers who have been developing a controller for an under-actuated crane system $^{[26]-[29]}$. Fundamentally, the HSMC approach enables engineers to design a control scheme based on hierarchical structure of sliding surfaces. In other words, first-order sliding surfaces are first formed by linear combination of state variables, which contribute different degrees of importance in the system performance, defined by their weights. Higher order sliding surfaces are then constructed to formulate a control law properly. For instance, in [26] Wang et $a l$. proposed a HSMC strategy for a class of under-actuated systems, where a second-order sliding surface is formed by linearly combining all firs-level sliding surfaces corresponding to all subsystems. By extending the method in [26], the authors of the work ${ }^{[30]}$ proposed another hierarchical structure of sliding surfaces in designing a control scheme for single-input-multiple-output under-actuated systems. They developed the first-layer sliding surface based on the first subsystem, which is then combined with the sliding surface of the second subsystem to form the second-layer sliding surface. This chain is subsequently conducted until the last subsystem. More importantly, the authors also proved that all the sliding surfaces in this hierarchical structure are stabilized. In [31], Qian et al. demonstrated feasibility of employing the HSMC law in an overhead crane system in a simulation environment.

Nonetheless, it is noted that the SMC strategy is mathematically deterministic, where all model parameters are required to be estimated. On the other hand, those parameters are also uncertain due to disturbances in the system. Some adaptive control strategies have been proposed to take the parameter uncertainties into account. For instance, in [32], [33], adaptive SMC schemes were developed for a crane system, where unknown parameters are estimated by an adaptive algorithm derived from Lyapunov theory. In the works [34], [35], a fuzzy uncertainty observer is employed to represent crane system uncertainties as well as actuator nonlinearities. Likewise, the SMC strategies were designed for non-linear systems in which unknown parameters are estimated by a neural network ${ }^{[36],[37]}$ and a fuzzy-neural network $^{[38]}$. Furthermore, an intelligent sliding mode controller comprising fuzzy wavelet neural networks for a 3D overhead crane is discussed in [39]. Though in the aforesaid works the approximation methods for estimating the uncertain parameters were validated in both simulations and laboratories, to the best of our knowledge, none of them was validated for a $3 \mathrm{D}$ model in a realistic crane system, where the microcontroller is resource-constrained. That is, 2D overhead cranes were considered in [34], [35] while a 3D model of an overhead crane was only simulated in [39].

Therefore, in this work, we propose a new efficient adaptive sliding mode control scheme for a $3 \mathrm{D}$ overhead crane system that comprises of hierarchical sliding surfaces and radial basis function networks (RBFN). In literature, the RBFN based SMC method has been employed in industry robotic systems [40], manipulators [41], [42], underactuated mechanical systems [43] and static var compensators [44]. The controller proposed in this paper is first created by the HSMC approach, where a second-level sliding surface is linearly constructed by two first-level sliding surfaces of two corresponding actuated and un-actuated subsystems. More particularly, nonlinear uncertain parameters of the system dynamics caused by external disturbances are presented and adaptively estimated by the use of the RBFN, which are then updated to the controller. The adaptive hierarchical sliding mode control (AHSMC) law was implemented and verified in both synthetic simulations and reallife experiments. The results obtained by the AHSMC are highly comparable to those obtained by HSMC. However, it is noticed that the proposed algorithm significantly reduces difficulties in determining unknown and uncertain parameters of a $3 \mathrm{D}$ overhead crane system. The results obtained by the proposed technique also demonstrate robustness of the system under uncertain conditions.

The remaining of the paper is organized as follows. Section 2 discusses a mathematical model of a 3D overhead crane while Section 3 presents the hierarchical sliding mode controller. The AHSMC strategy for a 3D overhead crane is then introduced in Section 4. Section 5 delineates the results and effectiveness of the proposed approach implemented in both synthetic simulations and real-life experiments before conclusions are drawn in Section 6 .

\section{3D Overhead Crane System}

\section{$2.1 \quad$ Notations}

Let $\mathbb{R}$ denote the set of real numbers. The Euclidean norm is defined by $\|\cdot\| \cdot \dot{y}$ and $\ddot{y}$ denote, respectively, the first and second derivatives of $y$. Moreover, we let $\operatorname{tr}(A)$ denote the trace of a matrix $A$. Other notations will be explained in due course.

\subsection{D Overhead Crane Model}

A 3D overhead crane can be delineated by a hoist motion in 3 directions ( $\mathrm{X}, \mathrm{Y}$ and $\mathrm{Z}$ ) in a Cartesian coordinate, which means it has three degrees of freedom ${ }^{[4]}$. Structurally, the crane consists of three main movable elements including a bridge, a trolley and a hoist. The bridge can move to the either left or right of a particular space. While the hoist can vertically lift cargo up and down, the trolley would carry it either backward or forward along the bridge.

Physically, a 3D overhead crane is represented by a model shown in Fig. 1. In a defined coordinate, positions of the bridge and trolley over time are specifically denoted by $x$ and $y$. While we let $\beta$ and $\alpha$ denote the angle between the hoist cable and its projection on the $\mathrm{X}-\mathrm{Z}$ plane and the angle between the projection of the hoist cable on the $\mathrm{X}-\mathrm{Z}$ plane and $\mathrm{Z}$ axis, respectively, length of the hoist cable is defined by $l$. Furthermore, it is assumed that the bridge is uniform, and the hoist cable is massless and rigid. If friction in the crane system is also supposed to be trivial, a dynamic model of a 3D overhead crane can be derived from the well-known Euler-Lagrange equation ${ }^{[45]}$ as follows,

$$
\frac{d}{d t}\left(\frac{\partial L}{\partial \dot{q}_{i}}\right)-\frac{\partial L}{\partial q_{i}}=\tau_{i}, i=1,2,3,4,5,
$$




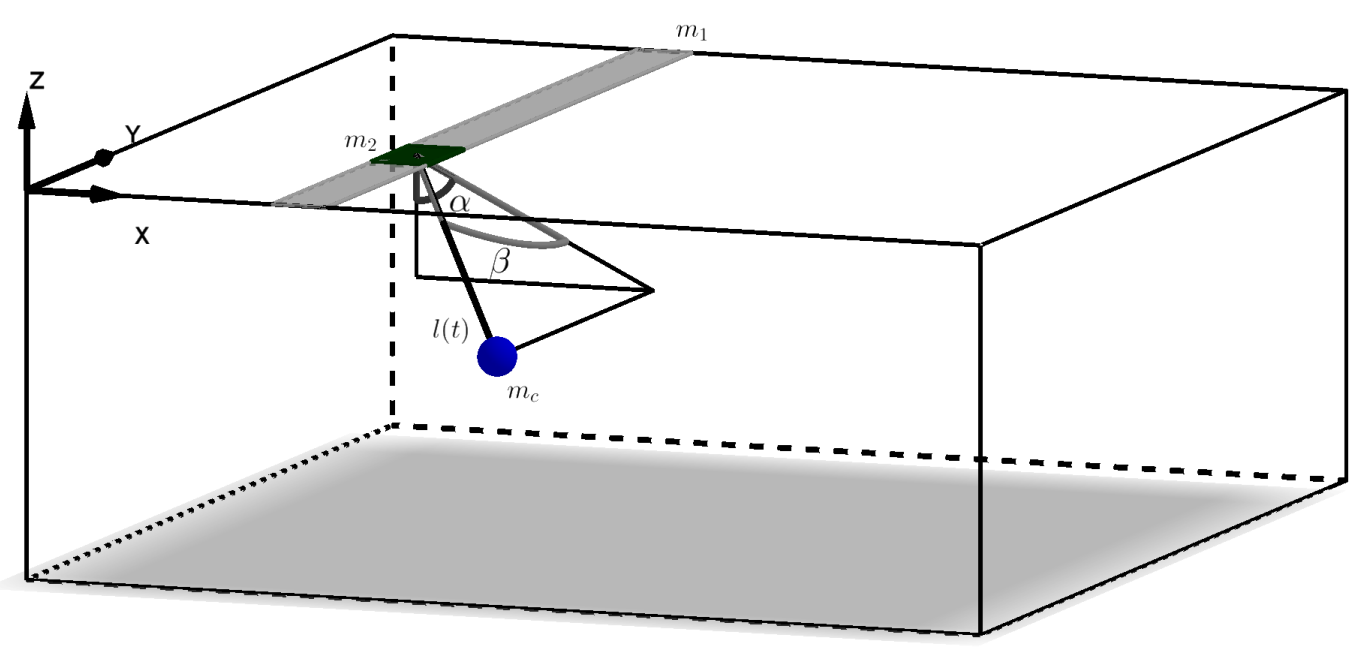

Figure 1: Modelling of a 3D overhead crane.

where $q_{i}$ is the $i^{\text {th }}$ element of the output vector $\mathbf{q}=$ $\left[\begin{array}{lllll}x & y & l & \alpha & \beta\end{array}\right]^{T}$ and $\tau_{i}$ is the $i^{\text {th }}$ control force of the input vector $\tau=\left[\begin{array}{lllll}\tau_{x} & \tau_{y} & \tau_{l} & 0 & 0\end{array}\right]^{T} . L=T-\Pi$, where $T$ and $\Pi$ are the kinetic and potential energies of the system. In the 3D overhead crane system, the kinetic and potential energies can be specified by

$$
\begin{aligned}
& T=\frac{1}{2} m_{b} \dot{x}^{2}+\frac{1}{2} m_{t}\left(\dot{x}^{2}+\dot{y}^{2}\right)+\frac{1}{2} m_{c}\left(\dot{x}_{c}^{2}+\dot{y}_{c}^{2}+\dot{z}_{c}^{2}\right), \\
& \Pi=m_{c} g z_{c}
\end{aligned}
$$

where $m_{b}$ and $m_{t}$ are the masses of the bridge and the trolley, respectively, $m_{c}$ is the mass of the cargo and $g=$ $9.8 \mathrm{~m} / \mathrm{s}^{2} . x_{c}, y_{c}$ and $z_{c}$ are the positions of the cargo, which can be obtained by

$$
\begin{aligned}
& x_{c}=x+l \cos \beta \sin \alpha \\
& y_{c}=y+l \sin \beta \\
& z_{c}=-l \cos \alpha \sin \beta .
\end{aligned}
$$

In the form of an ordinary differential equation system, the dynamic model of a 3D overhead crane can be systematically represented by

$$
\mathbf{M}(\mathbf{q}) \ddot{\mathbf{q}}+\mathbf{C}(\mathbf{q}, \dot{\mathbf{q}}) \dot{\mathbf{q}}+\mathbf{G}(\mathbf{q})=\tau
$$

where $\mathbf{M}(\mathbf{q}) \in \mathbb{R}^{5 \times 5}$ is the inertial matrix, $\mathbf{C}(\mathbf{q}, \dot{\mathbf{q}}) \in \mathbb{R}^{5 \times 5}$ is the centrifugal damping matrix, $\mathbf{G}(\mathbf{q}, \dot{\mathbf{q}}) \in \mathbb{R}^{5 \times 1}$ is the vector of the gravitational forces, and $\tau=\left[\begin{array}{lllll}\tau_{x} & \tau_{y} & \tau_{l} & 0 & 0\end{array}\right]^{T}$ is the vector of the control inputs. Elements of those matrices are computed as follows,

$$
\begin{aligned}
& m_{11}=m_{b}+m_{t}+m_{c}, m_{12}=m_{21}=0, \\
& m_{13}=m_{31}=m_{c} \sin \alpha \cos \beta, \\
& m_{14}=m_{41}=m_{c} l \cos \alpha \cos \beta, \\
& m_{15}=m_{51}=-m_{c} l \sin \alpha \sin \beta, \\
& m_{22}=m_{2}+m_{c}, m_{23}=m_{32}=m_{c} \sin \beta, \\
& m_{24}=m_{42}=0, m_{33}=m_{c}, m_{34}=m_{43}=0, \\
& m_{35}=m_{53}=0, m_{44}=m_{c} l^{2} \cos ^{2} \beta, \\
& m_{45}=m_{54}=0, m_{55}=m_{c} l^{2}, \\
& c_{11}=c_{12}=0, c_{13}=m_{c}(\dot{\alpha} \cos \alpha \cos \beta-\dot{\beta} \sin \alpha \sin \beta), \\
& c_{14}=-m_{c}(\dot{\alpha} l \cos \beta \sin \alpha+\dot{\beta} l \cos \alpha \sin \beta-i \cos \alpha \cos \beta), \\
& c_{21}=c_{22}=0, c_{23}=m_{c} \dot{\beta} \cos \beta, c_{24}=0, \\
& c_{25}=m_{c}(i \cos \beta-\dot{\beta} l \sin \beta), c_{31}=c_{32}=c_{33}=0, \\
& c_{34}=-m_{c} l \dot{\alpha} \cos { }^{2} \beta, c_{35}=-m_{c} l \dot{\beta}, c_{41}=c_{42}=0, \\
& c_{43}=m_{c} l \dot{\alpha} \cos \beta, c_{44}=m_{c} l \cos \beta(i \cos \beta-\dot{\beta} l \sin \beta), \\
& c_{45}=-m_{c} l^{2} \dot{\alpha} \sin \beta \cos \beta, c_{51}=c_{52}=0, c_{53}=m_{c} l \dot{\beta}, \\
& c_{54}=m_{c} l^{2} \dot{\alpha} \sin \beta \cos \beta, c_{55}=m_{c} l i, \\
& g_{1}=g_{2}=0, g_{3}=m_{c} g \cos \alpha \cos \beta, g_{4}=m_{c} g l \sin \alpha \cos \beta, \\
& g_{5}=-m_{c} g l \cos \alpha \sin \beta .
\end{aligned}
$$

\section{Hierarchical Sliding Mode Controller}

\subsection{Actuated and Un-actuated Decou- pling}

It is well-known challenging to design a controller for a $3 \mathrm{D}$ overhead crane system, which is under-actuated. In other words, of three outputs of the system $\mathbf{q}_{\mathbf{a}}=\left[\begin{array}{lll}x & y & l\end{array}\right]^{T}$ are directly controlled by its real three inputs $\tau_{\mathbf{1}}=\left[\begin{array}{lll}\tau_{x} & \tau_{y} & \tau_{l}\end{array}\right]^{T}$ 
correspondingly while two other outputs $\mathbf{q u}_{\mathbf{u}}=[\alpha \beta]^{T}$ are un-actuated. The un-actuated outputs can be only controlled through indirectly adjusting $x, y$ and $l$. Therefore, in order to facilitate the process of designing a controller, the dynamic model in (7) is separated into two equations and rewritten as follows,

$$
\begin{aligned}
\mathbf{M}_{11}(\mathbf{q}) \ddot{\mathbf{q}}_{\mathrm{a}}+\mathrm{M}_{12}(\mathbf{q}) \ddot{\mathbf{q}}_{\mathrm{u}}+\mathbf{C}_{11}(\mathbf{q}, \dot{\mathbf{q}}) \dot{\mathbf{q}}_{\mathrm{a}} \\
+\mathbf{C}_{12}(\mathbf{q}, \dot{\mathbf{q}}) \dot{\mathbf{q}}_{\mathrm{u}}+\mathbf{G}_{1}(\mathbf{q})=\tau_{1} \\
\mathbf{M}_{21}(\mathbf{q}) \ddot{\mathbf{q}}_{\mathrm{a}}+\mathbf{M}_{22}(\mathbf{q}) \ddot{\mathbf{q}}_{\mathrm{u}}+\mathbf{C}_{21}(\mathbf{q}, \dot{\mathbf{q}}) \dot{\mathbf{q}}_{\mathrm{a}} \\
+\mathbf{C}_{22}(\mathbf{q}, \dot{\mathbf{q}}) \dot{\mathbf{q}}_{\mathrm{u}}+\mathbf{G}_{2}(\mathbf{q})=\mathbf{0},
\end{aligned}
$$

where $\mathbf{M}_{\mathbf{1 1}}(\mathbf{q}) \in \mathbb{R}^{3 \times 3}, \mathbf{M}_{\mathbf{1 2}}(\mathbf{q}) \in \mathbb{R}^{3 \times 2}, \mathbf{M}_{\mathbf{2 1}}(\mathbf{q}) \in$ $\mathbb{R}^{2 \times 3}$ and $\mathbf{M}_{\mathbf{2 2}}(\mathbf{q}) \in \mathbb{R}^{2 \times 2}$ are sub-matrices of $\mathbf{M}(\mathbf{q})$. $\mathbf{C}_{1 \mathbf{1 1}}(\mathbf{q}, \dot{\mathbf{q}}) \in \mathbb{R}^{3 \times 3}, \quad \mathbf{C}_{\mathbf{1 2}}(\mathbf{q}, \dot{\mathbf{q}}) \in \mathbb{R}^{3 \times 2}, \mathbf{C}_{\mathbf{2 1}}(\mathbf{q}, \dot{\mathbf{q}}) \in$ $\mathbb{R}^{2 \times 3}$ and $\mathbf{C}_{\mathbf{2 2}}(\mathbf{q}, \dot{\mathbf{q}}) \in \mathbb{R}^{2 \times 2}$ are sub-matrices of $\mathbf{C}(\mathbf{q}, \dot{\mathbf{q}})$. $\mathbf{G}_{\mathbf{1}}(\mathbf{q}) \in \mathbb{R}^{3 \times 1}$ and $\mathbf{G}_{\mathbf{2}}(\mathbf{q}) \in \mathbb{R}^{2 \times 1}$ are sub-matrices of $\mathbf{G}(\mathbf{q})$, and $\mathbf{0}=\left[\begin{array}{ll}0 & 0\end{array}\right]^{T}$.

Since $\mathbf{M}_{\mathbf{2 2}}(\mathbf{q})$ is a positive definite matrix, (10) is rearranged as follows,

$$
\begin{aligned}
\ddot{\mathbf{q}}_{\mathrm{u}}=-\mathbf{M}_{22}^{-1}(\mathbf{q})\left[\mathbf{M}_{21}(\mathbf{q}) \ddot{\mathbf{q}}_{\mathbf{a}}\right. & +\mathbf{C}_{21}(\mathbf{q}, \dot{\mathbf{q}}) \dot{\mathbf{q}}_{\mathrm{a}} \\
& \left.+\mathbf{C}_{22}(\mathbf{q}, \dot{\mathbf{q}}) \dot{\mathbf{q}}_{\mathrm{u}}+\mathbf{G}_{2}(\mathbf{q})\right] .
\end{aligned}
$$

By substituting (12) into (8), the actuated outputs $\mathbf{q}_{\mathbf{a}}$ can be obtained by solving

$$
\overline{\mathbf{M}}_{\mathbf{1}}(\mathbf{q}) \ddot{\mathbf{q}}_{\mathbf{a}}+\overline{\mathbf{C}}_{11}(\mathbf{q}, \dot{\mathbf{q}}) \dot{\mathbf{q}}_{\mathbf{a}}+\overline{\mathbf{C}}_{\mathbf{1 2}}(\mathbf{q}, \dot{\mathbf{q}}) \dot{\mathbf{q}}_{\mathbf{u}}+\overline{\mathbf{G}}_{\mathbf{1}}(\mathbf{q})=\tau
$$

where

$$
\begin{aligned}
& \bar{M}_{1}(\mathbf{q})=\mathbf{M}_{11}(\mathbf{q})-\mathbf{M}_{12}(\mathbf{q}) \mathbf{M}_{22}^{-1}(\mathbf{q}) \mathbf{M}_{21}(\mathbf{q}) \\
& \overline{\mathbf{C}}_{11}(\mathbf{q}, \dot{\mathbf{q}})=\mathbf{C}_{11}(\mathbf{q}, \dot{\mathbf{q}})-\mathbf{M}_{12}(\mathbf{q}) \mathbf{M}_{22}^{-1}(\mathbf{q}) \mathbf{C}_{21}(\mathbf{q}, \dot{\mathbf{q}}) \\
& \overline{\mathbf{C}}_{12}(\mathbf{q}, \dot{\mathbf{q}})=\mathbf{C}_{12}(\mathbf{q}, \dot{\mathbf{q}})-\mathbf{M}_{12}(\mathbf{q}) \mathbf{M}_{22}^{-1}(\mathbf{q}) \mathbf{C}_{22}(\mathbf{q}, \dot{\mathbf{q}}) \\
& \overline{\mathbf{G}}_{1}(\mathbf{q})=\mathbf{G}_{1}(\mathbf{q})-\mathbf{M}_{12}(\mathbf{q}) \mathbf{M}_{22}^{-1}(\mathbf{q}) \mathbf{G}_{2}(\mathbf{q})
\end{aligned}
$$

Likewise, the un-actuated outputs $\mathbf{q u}_{\mathbf{u}}$ can be found by solving

$$
\overline{\mathbf{M}}_{2}(\mathbf{q}) \ddot{\mathbf{q}}_{\mathbf{a}}+\overline{\mathbf{C}}_{21}(\mathbf{q}, \dot{\mathbf{q}}) \dot{\mathbf{q}}_{\mathbf{a}}+\overline{\mathbf{C}}_{22}(\mathbf{q}, \dot{\mathbf{q}}) \dot{\mathbf{q}}_{\mathbf{u}}+\overline{\mathbf{G}}_{2}(\mathbf{q})=\overline{\mathbf{H}} \tau,
$$

where

$$
\begin{aligned}
& \overline{\mathbf{M}}_{2}(\mathbf{q})=\mathbf{M}_{22}(\mathbf{q})-\mathbf{M}_{21}(\mathbf{q}) \mathbf{M}_{11}^{-1}(\mathbf{q}) \mathbf{M}_{12}(\mathbf{q}) \\
& \overline{\mathbf{C}}_{21}(\mathbf{q}, \dot{\mathbf{q}})=\mathbf{C}_{21}(\mathbf{q}, \dot{\mathbf{q}})-\mathbf{M}_{21}(\mathbf{q}) \mathbf{M}_{11}^{-1}(\mathbf{q}) \mathbf{C}_{11}(\mathbf{q}, \dot{\mathbf{q}}) \\
& \overline{\mathbf{C}}_{22}(\mathbf{q}, \dot{\mathbf{q}})=\mathbf{C}_{22}(\mathbf{q}, \dot{\mathbf{q}})-\mathbf{M}_{21}(\mathbf{q}) \mathbf{M}_{11}^{-1}(\mathbf{q}) \mathbf{C}_{12}(\mathbf{q}, \dot{\mathbf{q}}) \\
& \overline{\mathbf{G}}_{2}(\mathbf{q})=\mathbf{G}_{2}(\mathbf{q})-\mathbf{M}_{21}(\mathbf{q}) \mathbf{M}_{11}^{-1}(\mathbf{q}) \mathbf{G}_{1}(\mathbf{q}) \\
& \overline{\mathbf{H}}=\mathbf{M}_{21}(\mathbf{q}) \mathbf{M}_{11}^{-1}(\mathbf{q}) .
\end{aligned}
$$

\subsection{Control Strategy}

In order to address the un-actuated control issues, many control schemes have been proposed for a 3D overhead crane system. Nevertheless, due to its renowned robustness under uncertainty conditions, sliding mode control (SMC) approach has remarkably attracted many crane control designers. Among the SMC designs, a hierarchical SMC ${ }^{[30]}$ presents to be effective for the control of an under-actuated system. In this section, we introduce a second-order sliding mode controller for a 3D overhead crane system, which will be then employed to develop its adaptive version in the following section.

Let $\mathbf{q}_{\mathbf{a d}}=\left[\begin{array}{lll}x_{d} & y_{d} & l_{d}\end{array}\right]^{T}$ and $\mathbf{q u d}_{\mathbf{u d}}=\left[\begin{array}{ll}0 & 0\end{array}\right]^{T}$ denote the output references for the system, to which the actuated states $\mathbf{q}_{\mathbf{a}}=$ $\left[\begin{array}{lll}x & y & l\end{array}\right]^{T}$ and un-actuated states $\mathbf{q}_{\mathbf{u}}=[\alpha \beta]^{T}$ are expected to converge, respectively. Thus, the corresponding errors are defined as follows,

$$
\begin{aligned}
& \mathbf{e}_{\mathbf{a}}=\mathbf{q}_{\mathbf{a}}-\mathbf{q}_{\mathbf{a d}} \\
& \mathbf{e}_{\mathbf{u}}=\mathbf{q}_{\mathbf{u}} .
\end{aligned}
$$

We first define two first-level sliding surfaces for the corresponding actuated and un-actuated subsystems as follows,

$$
\begin{aligned}
& \mathbf{s}_{1}=\dot{\mathbf{e}}_{\mathbf{a}}+\mathbf{c}_{\mathbf{a}} \mathbf{e}_{\mathbf{a}} \\
& \mathbf{s}_{2}=\dot{\mathbf{e}}_{\mathbf{u}}+\mathbf{c}_{\mathbf{u}} \mathbf{e}_{\mathbf{u}}
\end{aligned}
$$

where $\mathbf{s}_{\mathbf{1}} \in \mathbb{R}^{3 \times 1}, \mathbf{s}_{\mathbf{2}} \in \mathbb{R}^{3 \times 1}$, and $\mathbf{c}_{\mathbf{a}}=\operatorname{diag}\left(c_{1}, c_{2}, c_{3}\right)$ and $\mathbf{c}_{\mathbf{u}}=\operatorname{diag}\left(c_{4}, c_{5}\right)$ are positive constant parameters. The second-level sliding surface for the system is then aggregated by,

$$
\mathbf{s}=\mathbf{r}_{1} \mathbf{s}_{1}+\mathbf{r}_{2} \mathbf{s}_{2}
$$

where

$$
\begin{aligned}
& \mathbf{r}_{1}=\operatorname{diag}\left(r_{1}, r_{2}, r_{3}\right) \\
& \mathbf{r}_{2}=\left[\begin{array}{cc}
r_{4} & 0 \\
0 & r_{5} \\
0 & 0
\end{array}\right]
\end{aligned}
$$

are the matrices of positive gains. It is noticed that the second-level sliding surface $\mathbf{s} \in \mathbb{R}^{3}$ can be guaranteed to converge to zero if an appropriate control law is designed.

To this end, let us consider derivative of the second-level sliding surface. From equations (13) - (15) it derives that

$$
\begin{aligned}
& \dot{\mathrm{s}}=\mathrm{r}_{1} \dot{\mathrm{s}}_{1}+\mathrm{r}_{2} \dot{\mathrm{s}}_{2} \\
& =\mathbf{r}_{1}\left(\ddot{\mathbf{q}}_{\mathbf{a}}-\ddot{\mathbf{q}}_{\mathrm{ad}}\right)+\mathbf{r}_{1} \mathbf{c}_{\mathbf{a}}\left(\dot{\mathrm{q}}_{\mathbf{a}}-\dot{\mathbf{q}}_{\mathrm{ad}}\right)+\mathbf{r}_{2} \ddot{\mathbf{q}}_{\mathbf{u}}+\mathbf{r}_{2} \mathbf{c}_{\mathbf{u}} \dot{\mathbf{q}}_{\mathbf{u}} \\
& =\left[\begin{array}{l}
\mathbf{r}_{1} \overline{\mathbf{M}}_{1}^{-1}\left(\tau-\mathbf{C}_{11} \dot{\mathbf{q}}_{\mathrm{a}}-\mathbf{C}_{12} \dot{\mathbf{q}}_{\mathrm{u}}-\overline{\mathbf{G}}_{1}\right)-\mathbf{r}_{1} \ddot{\mathbf{q}}_{\mathrm{ad}} \\
+\mathbf{r}_{1} \mathbf{c}_{\mathrm{a}} \dot{\mathbf{q}}_{\mathrm{a}}-\mathbf{r}_{1} \mathbf{c}_{\mathrm{a}} \dot{\mathbf{q}}_{\mathrm{ad}} \\
+\mathbf{r}_{2} \overline{\mathbf{M}}_{2}^{-1}\left(\overline{\mathbf{H}} \tau-\mathbf{C}_{21} \dot{\mathbf{q}}_{\mathrm{a}}-\mathbf{C}_{22} \dot{\mathbf{q}}_{\mathrm{u}}-\overline{\mathbf{G}}_{2}\right)+\mathbf{r}_{2} \mathbf{c}_{\mathrm{u}} \dot{\mathbf{q}}_{\mathrm{u}}
\end{array}\right] .
\end{aligned}
$$

If we differentiate the Lyapunov function candidate $V=$ $\frac{1}{2} \mathbf{S}^{T} \mathbf{S}$ wrt time, and given the derivative of the second-level sliding surface in (16), it yields

$$
\begin{aligned}
\dot{V} & =\dot{\mathrm{s}}^{T} \mathbf{s} \\
& =\left[\begin{array}{l}
\left(\mathbf{r}_{1} \overline{\mathbf{M}}_{1}^{-1}+\mathbf{r}_{2} \overline{\mathbf{M}}_{2}^{-1} \overline{\mathbf{H}}_{\tau}\right) \\
-\mathbf{r}_{1} \overline{\mathbf{M}}_{1}^{-1}\left(\mathbf{C}_{11} \dot{\mathbf{q}}_{\mathbf{a}}+\mathbf{C}_{12} \dot{\mathbf{q}}_{\mathbf{u}}+\overline{\mathbf{G}}_{1}\right) \\
-\mathbf{r}_{1} \ddot{\mathbf{q}}_{\mathbf{a d}}+\mathbf{r}_{1} \mathbf{c}_{\mathbf{a}} \dot{\mathbf{q}}_{\mathbf{a}}-\mathbf{r}_{1} \mathbf{c}_{\mathbf{a}} \dot{\mathbf{q}}_{\mathbf{a d}} \\
-\mathbf{r}_{2} \overline{\mathbf{M}}_{2}^{-1}\left(\mathbf{C}_{21} \dot{\mathbf{q}}_{\mathbf{a}}+\mathbf{C}_{22} \dot{\mathbf{q}}_{\mathbf{u}}+\overline{\mathbf{G}}_{2}\right)+\mathbf{r}_{2} \mathbf{c}_{\mathbf{u}} \dot{\mathbf{q}}_{\mathbf{u}}
\end{array}\right]^{T} \mathbf{s} .
\end{aligned}
$$

In order to stabilize the second-level sliding surface $\mathbf{s}$, we let

$$
\dot{\mathbf{s}}+\mathbf{K}_{\mathbf{1}} \operatorname{sgn}(\mathbf{s})+\mathbf{K}_{\mathbf{2}} \mathbf{s}=\mathbf{0},
$$

where $\mathbf{K}_{\mathbf{1}}=\operatorname{diag}\left(K_{11}, K_{12}, K_{13}\right)$ and $\mathbf{K}_{\mathbf{2}}=$ $\operatorname{diag}\left(K_{21}, K_{22}, K_{23}\right)$ are the positive control gain matrices. Note that $\mathbf{K}_{\mathbf{1}}$ and $\mathbf{K}_{\mathbf{2}}$ are chosen so that stability of 


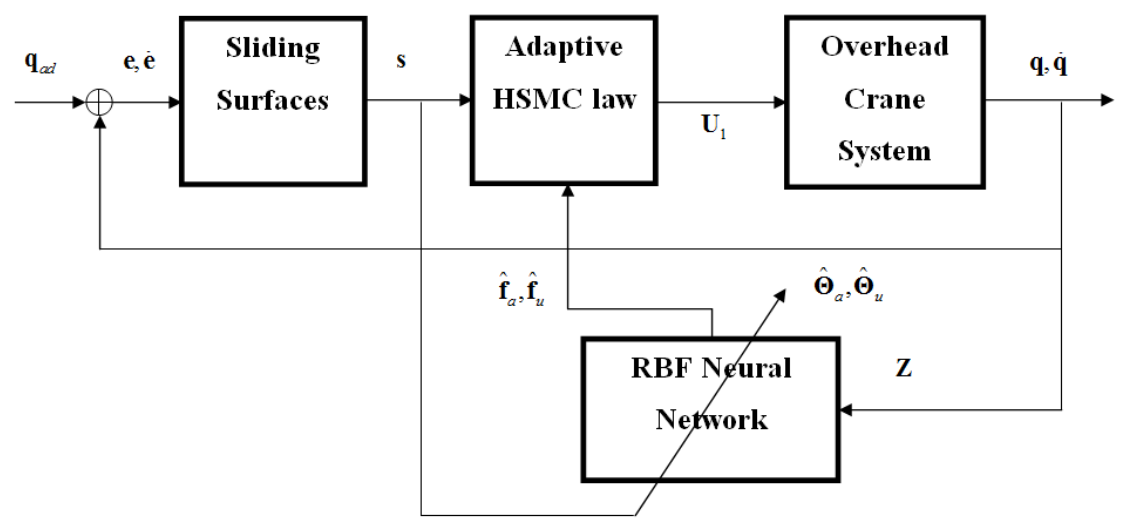

Figure 2: The diagram of the HSMC scheme.

the sliding surface is guaranteed ${ }^{[21]}$. Therefore, the HSMC scheme for a $3 \mathrm{D}$ overhead crane can be presented as follows

$$
\begin{aligned}
\tau= & {\left[\mathbf{r}_{1} \overline{\mathbf{M}}_{1}^{-1}+\mathbf{r}_{2} \overline{\mathbf{M}}_{2}^{-1} \overline{\mathbf{H}}\right]^{-1} \times } \\
& \times\left[\begin{array}{l}
\mathbf{r}_{1} \overline{\mathbf{M}}_{1}^{-1}\left(\mathbf{C}_{11} \dot{\mathbf{q}}_{\mathbf{a}}+\mathbf{C}_{12} \dot{\mathbf{q}}_{\mathbf{u}}+\overline{\mathbf{G}}_{1}\right) \\
-\left(-\mathbf{r}_{1} \ddot{\mathbf{q}}_{\mathbf{a d}}+\mathbf{r}_{1} \mathbf{c}_{\mathrm{a}} \dot{\mathbf{q}}_{\mathbf{a}}-\mathbf{r}_{1} \mathbf{c}_{\mathrm{a}} \dot{\mathbf{q}}_{\mathbf{a d}}\right. \\
+\mathbf{r}_{2} \overline{\mathbf{M}}_{2}^{-1}\left(\mathbf{C}_{21} \dot{\mathbf{q}}_{\mathbf{a}}+\mathbf{C}_{22} \dot{\mathbf{q}}_{\mathbf{u}}+\overline{\mathbf{G}}_{2}\right) \\
-\mathbf{r}_{2} \mathbf{c}_{\mathbf{u}} \dot{\mathbf{q}}_{\mathbf{u}}-\mathbf{K}_{1} \operatorname{sgn}(\mathbf{s})-\mathbf{K}_{2} \mathbf{s}
\end{array}\right] .
\end{aligned}
$$

Nonetheless, due to the "chattering" phenomenon, which may practically affect on the system trajectories that are consistently expected to be maintained on the sliding surface, a saturation function $\operatorname{sat}(\cdot)$ is proposed to replace the switching function $\operatorname{sgn}(\cdot)$ in (18). In this work, the saturation function $\operatorname{sat}(\cdot)$ is defined as follows,

$$
\operatorname{sat}(x / b)= \begin{cases}-1, & \text { if } x \leq-b \\ x / b, & \text { if }-b \leq x \leq b \\ 1 & \text { if } x \geq b,\end{cases}
$$

where $b$ is a parameter that can be chosen by the trial-anderror method so that all of the chattering will be eliminated given different frequency bands.

\section{Adaptive Hierarchical Sliding Mode Control Design}

\subsection{Neural Network based Controller}

The HSMC strategy in (18) is mathematically deterministic, which means that this control scheme can only be practical if all the parameters involved in the system model are known. Nonetheless, determining some of those parameters is not trivial in a realistic application. On the other hand, uncertainties of the model parameters are not considered in (18). Moreover, some other nonlinear characteristics such as the dead-zone band of actuators ${ }^{[46]}$ and the hysteresis of outputs ${ }^{[4]}$ are disregarded in the deterministic model. Therefore, the deterministic HSMC law is practically demolished.

To effectively deal with the aforesaid limitations of the deterministic model, we propose to exploit a Radial Basis
Function Network (RBFN) to adaptively estimate the parameters in the control strategy for a $3 \mathrm{D}$ overhead crane system. Since the RBFN is capable of processing nonlinear features in a system, both the parameter uncertainties and external disturbances in the system are well incorporated into the dynamics in the adaptive hierarchical sliding mode controller obtained by this learning mechanism. The structure of the HSMC law is illustrated in Fig. 2

In the context of a feedforward neural network, as discussed by Park et al. in [48], an $N$ node RBFN based universal approximation can be represented by

$$
f\left(\mathbf{Z}=\sum_{i=1}^{N} \omega_{i} s_{i}(\mathbf{Z})=\mathbf{W}^{\mathbf{T}} \mathbf{S}(\mathbf{Z}),\right.
$$

where $s_{i}(\cdot)$ is a radial basis function $(\mathrm{RBF}), \mathbf{Z}$ is the input vector, $\mathbf{W}=\left[\omega_{1}, \cdots, \omega_{N}\right]^{T} \in \mathbb{R}^{N}$ is the weight vector and $\mathbf{S}(\mathbf{Z})=\left[s_{1}\left(\left\|\mathbf{Z}-\mu_{\mathbf{1}}\right\|\right), \cdots, s_{N}\left(\left\|\mathbf{Z}-\mu_{\mathbf{1}}\right\|\right)\right]^{T}$ is considered as a set of activation functions. In fact, there is a family of $\mathrm{RBFs}^{[49]}$, and the widely used Gaussian function is specified as follows,

$$
s_{i}\left(\left\|\mathbf{Z}-\mu_{\mathbf{1}}\right\|\right)=\exp \left(-\frac{\left(\mathbf{Z}-\mu_{\mathbf{i}}\right)^{T}\left(\mathbf{Z}-\mu_{\mathbf{i}}\right)}{2 \delta_{i}^{2}}\right),
$$

where $\mu_{\mathbf{i}}=\left[\mu_{i 1}, \cdots \mu_{i q}\right]^{T}$ is the centre vector and $\delta_{i}$ is the standard deviation.

It is noted that if the number of nodes $N$ is large enough, a RBFN can be approximately presented by a continuous function. In other words, in steps of designing a control law for a 3D overhead crane, a RBFN can be intelligently exploited to model some unknown dynamics in the system. To this end, the HSMC strategy in (18) can be rewritten by

$$
\begin{aligned}
\tau & =\left[\mathbf{r}_{1} \overline{\mathbf{M}}_{1}^{-1}+\mathbf{r}_{2} \overline{\mathbf{M}}_{2}^{-1} \overline{\mathbf{H}}\right]^{-1} \times \\
& \times\left[\begin{array}{c}
\mathbf{r}_{1} \overline{\mathbf{M}}_{1}^{-1} \mathbf{f}_{\mathbf{a}}(\mathbf{q}, \dot{\mathbf{q}})-\left(-\mathbf{r}_{1} \ddot{\mathbf{q}}_{\mathbf{a d}}+\mathbf{r}_{1} \mathbf{c}_{\mathbf{a}} \dot{\mathbf{q}}_{\mathbf{a}}-\mathbf{r}_{1} \mathbf{c}_{\mathbf{a}} \dot{\mathbf{q}}_{\mathbf{a d}}\right. \\
+\mathbf{r}_{2} \overline{\mathbf{M}}_{2}^{-1} \mathbf{f}_{\mathbf{u}}(\mathbf{q}, \dot{\mathbf{q}})-\mathbf{r}_{2} \mathbf{c}_{\mathbf{u}} \dot{\mathbf{q}}_{\mathbf{u}}-\mathbf{K}_{\mathbf{1}} \operatorname{sgn}(\mathbf{s})-\mathbf{K}_{2} \mathbf{s}
\end{array}\right],
\end{aligned}
$$

where

$$
\begin{aligned}
& \mathbf{f}_{\mathrm{a}}(\mathbf{q}, \dot{\mathbf{q}})=\overline{\mathbf{C}}_{11}(\mathbf{q}, \dot{\mathbf{q}}) \dot{\mathbf{q}}_{\mathrm{a}} \overline{\mathbf{C}}_{12}(\mathbf{q}, \dot{\mathbf{q}}) \dot{\mathbf{q}}_{\mathrm{u}}+\overline{\mathbf{G}}_{1}(\mathbf{q}) \\
& \mathrm{f}_{\mathrm{u}}(\mathbf{q}, \dot{\mathbf{q}})=\overline{\mathbf{C}}_{21}(\mathbf{q}, \dot{\mathbf{q}}) \dot{\mathbf{q}}_{\mathrm{a}} \overline{\mathbf{C}}_{22}(\mathbf{q}, \dot{\mathbf{q}}) \dot{\mathbf{q}}_{\mathrm{u}}+\overline{\mathbf{G}}_{2}(\mathbf{q})
\end{aligned}
$$


are the continuous functions corresponding to the actuated and un-actuated outputs. If we define the state inputs of a RBFN as $\mathbf{Z}=\left[\mathbf{e}_{\mathbf{s t}} \mathbf{e}_{\mathbf{s t}}\right]^{T} \in \mathbb{R}^{6}$ with $\mathbf{e}_{\mathbf{s t}}=\left[\begin{array}{lll}l & \alpha \beta\end{array}\right]^{T} \in \mathbb{R}^{3}$, let $\hat{\mathbf{f}}_{\mathbf{a}}(\mathbf{Z}) \in \mathbb{R}^{3}$ and $\hat{\mathbf{f}}_{\mathbf{u}}(\mathbf{Z}) \in \mathbb{R}^{2}$ denote the corresponding approximations of $\mathbf{f}_{\mathbf{a}}(\mathbf{q}, \dot{\mathbf{q}})$ and $\mathbf{f}_{\mathbf{u}}(\mathbf{q}, \dot{\mathbf{q}})$, which are obtained by the RBFNs and given as follows,

$$
\begin{aligned}
& \hat{\mathbf{f}}_{\mathbf{a}}(\mathbf{Z})=\hat{\mathbf{W}}_{\mathbf{a}}^{\mathbf{T}} \mathbf{S}(\mathbf{Z}) \\
& \hat{\mathbf{f}}_{\mathbf{u}}(\mathbf{Z})=\hat{\mathbf{W}}_{\mathbf{u}}^{\mathbf{T}} \mathbf{S}(\mathbf{Z}),
\end{aligned}
$$

where $\hat{\mathbf{W}}_{\mathbf{a}}^{\mathbf{T}} \in \mathbb{R}^{\mathbf{3} \times \mathbf{N}}$ and $\hat{\mathbf{W}}_{\mathbf{u}}^{\mathbf{T}} \in \mathbb{R}^{\mathbf{2} \times \mathbf{N}}$ are the adaptive weight matrices, which can be obtained by the use of the Lyapunov stability and presented by

$$
\begin{aligned}
& \dot{\hat{\mathbf{W}}}_{\mathrm{a}}=-\Gamma_{\mathrm{a}} \mathbf{S}(\mathbf{Z}) \mathbf{s}^{\mathrm{T}} \mathbf{r}_{1} \overline{\mathbf{M}}_{1}^{-1} \\
& \dot{\hat{\mathbf{W}}}_{\mathbf{u}}=-\Gamma_{\mathrm{u}} \mathbf{S}(\mathbf{Z}) \mathbf{s}^{\mathrm{T}} \mathbf{r}_{2} \overline{\mathbf{M}}_{2}^{-1},
\end{aligned}
$$

where $\boldsymbol{\Gamma}_{\mathbf{a}}=\operatorname{diag}\left(\Gamma_{a 1}, \Gamma_{a 2}, \cdots, \Gamma_{a N}\right)$ and $\boldsymbol{\Gamma}_{\mathbf{u}}=$ $\operatorname{diag}\left(\Gamma_{u 1}, \Gamma_{u 2}, \cdots, \Gamma_{u N}\right)$ are positive definite matrices. Therefore, the HSMC scheme (21) is adaptively approximated by

$$
\begin{aligned}
\tau & =\left[\mathbf{r}_{1} \overline{\mathbf{M}}_{1}^{-1}+\mathbf{r}_{\mathbf{2}} \overline{\mathbf{M}}_{2}^{-1} \overline{\mathbf{H}}\right]^{-1} \times \\
& \times\left[\begin{array}{c}
\mathbf{r}_{1} \overline{\mathbf{M}}_{1}^{-1} \hat{\mathbf{f}}_{\mathbf{a}}(\mathbf{q}, \dot{\mathbf{q}})-\left(-\mathbf{r}_{1} \ddot{\mathbf{q}}_{\mathbf{a d}}+\mathbf{r}_{\mathbf{1}} \mathbf{c}_{\mathbf{a}} \dot{\mathbf{q}}_{\mathbf{a}}-\mathbf{r}_{1} \mathbf{c}_{\mathbf{a}} \dot{\mathbf{q}}_{\mathbf{a d}}\right. \\
+\mathbf{r}_{2} \overline{\mathbf{M}}_{\mathbf{2}}^{-\mathbf{1}} \hat{\mathbf{f}}_{\mathbf{u}}(\mathbf{q}, \dot{\mathbf{q}})-\mathbf{r}_{\mathbf{2}} \mathbf{c}_{\mathbf{u}} \dot{\mathbf{q}}_{\mathbf{u}}-\mathbf{K}_{\mathbf{1}} \operatorname{sgn}(\mathbf{s})-\mathbf{K}_{\mathbf{2}} \mathbf{s}
\end{array}\right] .
\end{aligned}
$$

\subsection{Sliding Surface Stability Analysis}

In order to demonstrate effectiveness of the proposed adaptive HSMC (AHSMC) scheme (24) for a 3D overhead crane, this section discusses stability of the closed-loop control system. Lets consider the Lyapunov function candidate, specified by

$$
V=\frac{1}{2} \mathbf{s}^{\mathbf{T}} \mathbf{s}+\frac{1}{2} \operatorname{tr}\left(\tilde{\mathbf{W}}_{\mathbf{a}}^{\mathbf{T}} \boldsymbol{\Gamma}_{\mathbf{a}}^{-1} \tilde{\mathbf{W}}_{\mathbf{a}}\right)+\frac{1}{2} \operatorname{tr}\left(\tilde{\mathbf{W}}_{\mathbf{u}}^{\mathbf{T}} \boldsymbol{\Gamma}_{\mathbf{u}}^{-1} \tilde{\mathbf{W}}_{\mathbf{u}}\right),
$$

where $\tilde{\mathbf{W}}_{\mathbf{a}}=\hat{\mathbf{W}}_{\mathbf{a}}-\mathbf{W}_{\mathbf{a}}$ and $\tilde{\mathbf{W}}_{\mathbf{u}}=\hat{\mathbf{W}}_{\mathbf{u}}-\mathbf{W}_{\mathbf{u}}$ are the weight errors. And its derivative wrt time is in the form of

$$
\begin{aligned}
& \dot{V}=\mathbf{s}^{\mathbf{T}} \dot{\mathbf{s}}+\operatorname{tr}\left(\tilde{\mathbf{W}}_{\mathbf{a}}^{\mathbf{T}} \boldsymbol{\Gamma}_{\mathbf{a}}^{-1} \dot{\hat{\mathbf{W}}}_{\mathbf{a}}\right)+\operatorname{tr}\left(\tilde{\mathbf{W}}_{\mathbf{u}}^{\mathbf{T}} \boldsymbol{\Gamma}_{\mathbf{u}}^{-1} \dot{\hat{\mathbf{W}}}_{\mathbf{u}}\right) \\
& =\left[\begin{array}{l}
\mathbf{s}^{T}\left(\left(\mathbf{r}_{1} \overline{\mathbf{M}}_{1}^{-1}+\mathbf{r}_{\mathbf{2}} \overline{\mathbf{M}}_{\mathbf{2}}^{-1} \overline{\mathbf{H}}\right) \tau-\mathbf{r}_{1} \overline{\mathbf{M}}_{\mathbf{1}}^{-1} \mathbf{f}_{\mathbf{a}}\right. \\
-\mathbf{r}_{1} \ddot{\mathbf{q}}_{\mathbf{a d}}+\mathbf{r}_{1} \mathbf{c}_{\mathbf{a}} \dot{\mathbf{q}}_{\mathbf{a}}-\mathbf{r}_{1} \mathbf{c}_{\mathbf{a}} \dot{\mathbf{q}}_{\mathbf{a d}}-\mathbf{r}_{\mathbf{2}} \overline{\mathbf{M}}_{\mathbf{2}}^{-1} \mathbf{f}_{\mathbf{u}} \\
\left.+\mathbf{r}_{\mathbf{2}} \mathbf{c}_{\mathbf{u}} \dot{\mathbf{q}}_{\mathbf{u}}\right)+\operatorname{tr}\left(\tilde{\mathbf{W}}_{\mathbf{a}}^{\mathbf{T}} \boldsymbol{\Gamma}_{\mathbf{a}}^{-1} \dot{\hat{\mathbf{W}}}_{\mathbf{a}}\right)+\operatorname{tr}\left(\tilde{\mathbf{W}}_{\mathbf{u}}^{\mathbf{T}} \boldsymbol{\Gamma}_{\mathbf{u}}^{-1} \dot{\hat{\mathbf{W}}}_{\mathbf{u}}\right)
\end{array}\right] \\
& =\left[\begin{array}{l}
\mathbf{s}^{T}\left(\left(\mathbf{r}_{1} \overline{\mathbf{M}}_{1}^{-1}+\mathbf{r}_{2} \overline{\mathbf{M}}_{2}^{-1} \overline{\mathbf{H}}\right) \tau-\mathbf{r}_{1} \overline{\mathbf{M}}_{1}^{-1} \hat{\mathbf{f}}_{\mathbf{a}}\right. \\
-\mathbf{r}_{1} \ddot{\mathbf{q}}_{\mathbf{a d}}+\mathbf{r}_{1} \mathbf{c}_{\mathbf{a}} \dot{\mathbf{q}}_{\mathbf{a}}-\mathbf{r}_{1} \mathbf{c}_{\mathbf{a}} \dot{\mathbf{q}}_{\mathbf{a d}}-\mathbf{r}_{2} \overline{\mathbf{M}}_{2}^{-1} \hat{\mathbf{f}}_{\mathbf{u}} \\
\left.+\mathbf{r}_{2} \mathbf{c}_{\mathbf{u}} \dot{\mathbf{q}}_{\mathbf{u}}\right)+\mathbf{s}^{\mathbf{T}} \mathbf{r}_{1} \overline{\mathbf{M}}_{1}^{-1}\left(\mathbf{f}_{\mathbf{a}}-\hat{\mathbf{f}}_{\mathbf{a}}\right) \\
+\mathbf{s}^{\mathbf{T}} \mathbf{r}_{2} \overline{\mathbf{M}}_{2}^{-1}\left(\mathbf{f}_{\mathbf{u}}-\hat{\mathbf{f}}_{\mathbf{u}}\right)+\operatorname{tr}\left(\tilde{\mathbf{W}}_{\mathbf{a}}^{\mathbf{T}} \boldsymbol{\Gamma}_{\mathbf{a}}^{-1} \dot{\hat{\mathbf{W}}}_{\mathbf{a}}\right) \\
+\operatorname{tr}\left(\tilde{\mathbf{W}}_{\mathbf{u}}^{\mathbf{T}} \mathbf{\Gamma}_{\mathbf{u}}^{-1} \dot{\hat{\mathbf{W}}}_{\mathbf{u}}\right)
\end{array}\right] .
\end{aligned}
$$

Substituting the AHSMC law (24) into (26) yields

$$
\begin{aligned}
\dot{V} & =-\mathbf{s}^{\mathbf{T}} \mathbf{K}_{\mathbf{1}} \operatorname{sgn}(\mathbf{s})-\mathbf{s}^{\mathbf{T}} \mathbf{K}_{\mathbf{2}} \mathbf{S}+\mathbf{s}^{\mathbf{T}} \mathbf{r}_{\mathbf{1}} \mathbf{M}_{\mathbf{1}}^{-1} \tilde{\mathbf{W}}_{\mathbf{a}}^{\mathbf{T}} \mathbf{S}(\mathbf{Z}) \\
& +\mathbf{s}^{\mathbf{T}} \mathbf{r}_{\mathbf{1}} \mathbf{M}_{\mathbf{1}}^{-\mathbf{1}} \tilde{\mathbf{W}}_{\mathbf{u}}^{\mathbf{T}} \mathbf{S}(\mathbf{Z})+\operatorname{tr}\left(\tilde{\mathbf{W}}_{\mathbf{a}}^{\mathbf{T}} \boldsymbol{\Gamma}_{\mathbf{a}}^{-\mathbf{1}} \dot{\hat{\mathbf{W}}}_{\mathbf{a}}\right) \\
& +\operatorname{tr}\left(\tilde{\mathbf{W}}_{\mathbf{u}}^{\mathbf{T}} \boldsymbol{\Gamma}_{\mathbf{u}}^{-\mathbf{1}} \dot{\hat{\mathbf{W}}}_{\mathbf{u}}\right) .
\end{aligned}
$$

Moreover, (27) can be further simplified by using the adaptive mechanisms in (22) and (23) as follows,

$$
\dot{V}=-\mathbf{s}^{\mathbf{T}} \mathbf{K}_{1} \operatorname{sgn}(\mathbf{s})-\mathbf{s}^{\mathbf{T}} \mathbf{K}_{2} \mathbf{s} \leq 0 .
$$

Integrating (28) yields

$$
\int_{0}^{t} d V=\int_{0}^{t}\left(-\mathbf{K}_{\mathbf{2}} \mathbf{s}^{\mathbf{T}} \mathbf{s}-\mathbf{K}_{\mathbf{1}}|\mathbf{s}|\right) d t=V(t)-V(0) .
$$

Hence,

$V(t)=\frac{1}{2} \mathbf{s}^{\mathbf{T}} \mathbf{s}+\frac{1}{2} \operatorname{tr}\left(\tilde{\mathbf{W}}_{\mathbf{a}}^{\mathbf{T}} \boldsymbol{\Gamma}_{\mathbf{a}}^{-1} \tilde{\mathbf{W}}_{\mathbf{a}}\right)+\frac{1}{2} \operatorname{tr}\left(\tilde{\mathbf{W}}_{\mathbf{u}}^{\mathbf{T}} \boldsymbol{\Gamma}_{\mathbf{u}}^{-1} \tilde{\mathbf{W}}_{\mathbf{u}}\right) \leq V(0)$,

which means $\mathbf{s}, \tilde{\mathbf{W}}_{\mathbf{a}}$ and $\tilde{\mathbf{W}}_{\mathbf{u}}$ are bounded. Furthermore, since $V(0) \geq 0$,

$$
\int_{0}^{t}\left(\mathbf{K}_{\mathbf{2}} \mathbf{s}^{\mathbf{T}} \mathbf{s}-\mathbf{K}_{\mathbf{1}}|\mathbf{s}|\right) d t \leq 0 .
$$

Thus, $\lim _{t \rightarrow \infty} \mathbf{s}=\mathbf{0}$; that is, the second-level sliding surface $\mathbf{s}$ in (15) is asymptotically stabilized.

\subsection{Actuator Saturation}

In practice, actuators of a crane system have nonlinear constraints such as backlash, dead-zone band, hysteresis of outputs or saturation features. In other words, the control signals may cause actuations to be exceeding a safety margin, which mitigates quality of the control law and destroys the actuators. To address this issue, we have taken the actuator saturation into account. The saturation of the actuators is defined as follows [50].

$$
\tau_{s}= \begin{cases}\tau_{\max } & \text { if } \tau>\tau_{\max } \\ \tau & \text { if } \tau_{\min }<\tau<\tau_{\max } \\ \tau_{\min } & \text { if } \tau<\tau_{\min },\end{cases}
$$

where $\tau_{s}$ is the actuator output and $\tau$ is the control input of the actuator. $\tau_{\max }$ and $\tau_{\min }$ are the upper and lower bounds of the actuator characteristics, respectively. If the control input $\tau$ is outside the linear range of the actuator, there exists the saturation nonlinearity and the calculated control signal cannot effectively control the object. Thus, the eliminated term of the control signal can be recomputed by

$$
\mathbf{M}(\mathbf{q}) \ddot{\mathbf{q}}+\mathbf{C}(\mathbf{q}, \dot{\mathbf{q}}) \dot{\mathbf{q}}+\mathbf{G}(\mathbf{q})=\tau_{\mathbf{s}}+\delta
$$

where $\delta=\tau-\tau_{\mathbf{s}}$ represents influence of the saturation features. In cases, where effect of the actuator saturation on system is considered, the control signal $\tau$ in (21) is replaced by $\tau_{s}$ in (32).

\section{$5 \quad$ Results and Discussions}

In order to illustrate effectiveness of the proposed approach of the AHSMC as compared with that of the HSMC, this section discusses results obtained in the experiments conducted on both a synthetic simulation environment and in a laboratory. The aim was to investigate how efficiently the responses of the bridge, trolley, hoist and hoist cable converge to desired steady states after each control action. It is noted that the RBFN exploited in all the experiments was constructed by 100 nodes, where their centres were evenly distributed in $\mu_{\mathbf{i}}=[-1.2,1.2] \times[-1.6,1.6]$ and the standard deviation was set to $\delta_{i}=0.8$, for $i=1,2, \cdots, 6$. 


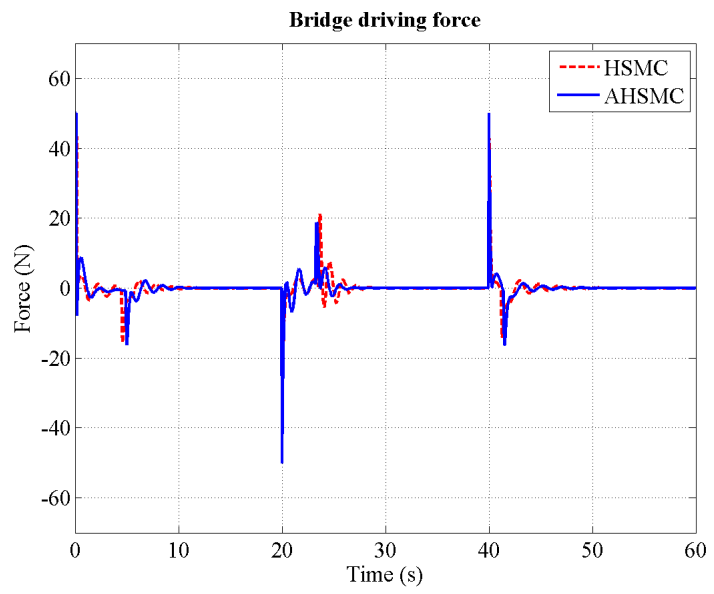

(a)

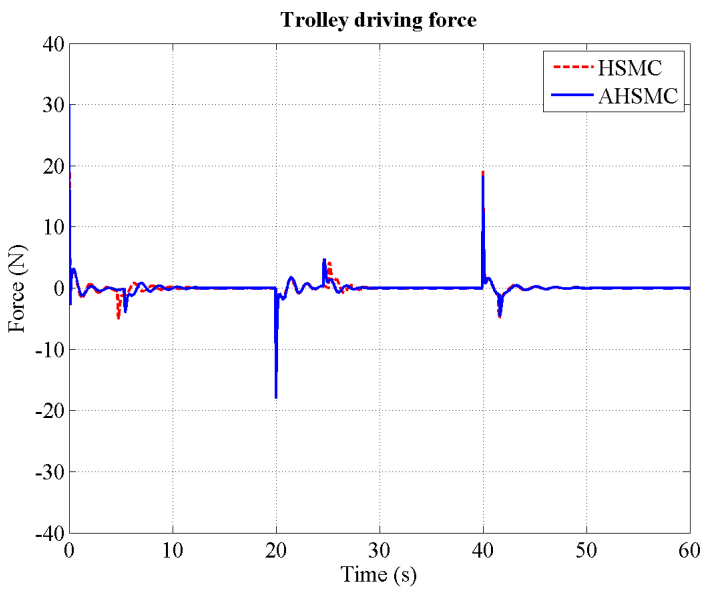

(b)

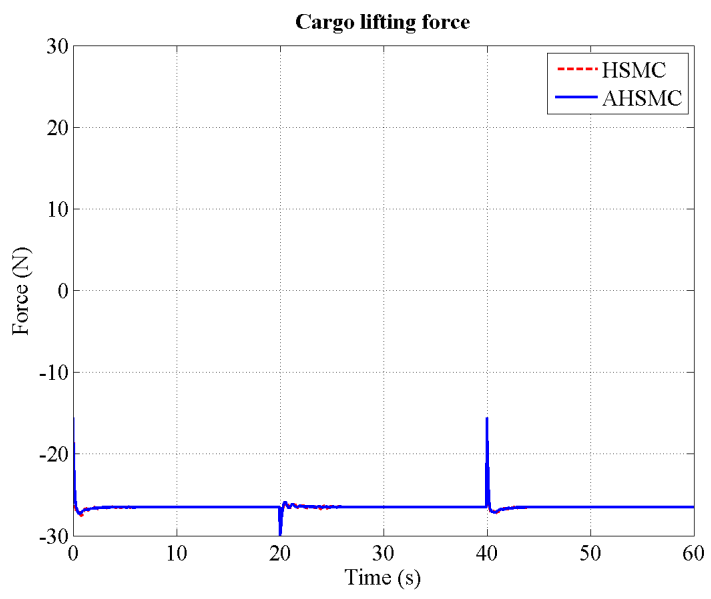

(c)

Figure 3: Simulation control force signals.

\subsection{Simulations}

In the first experiments, we synthetically simulated a real-life 3D overhead crane system that is pictorially shown in Fig. 6. Therefore, information of the bridge crane was known. Furthermore, parameters of the controllers were given. The parameters of the $3 \mathrm{D}$ overhead crane system and its controllers including the HSMC and AHSMC are summarized as follows

$$
\begin{aligned}
& m_{b}=24.3 \mathrm{~kg}, m_{t}=11.2 \mathrm{~kg}, \\
& c_{1}=c_{3}=4, c_{2}=5, c_{4}=c_{5}=0.02 \text {, } \\
& m_{c}=0.78 \mathrm{~kg}, g=9.81 \mathrm{~m} / \mathrm{s}^{2} \text {, } \\
& \tau_{\max }=\left[\begin{array}{lllll}
60 & 30 & 30 & 0 & 0
\end{array}\right], \tau_{\min }=\left[\begin{array}{lllll}
-60 & -30 & -30 & 0 & 0
\end{array}\right], \\
& \mathbf{q}(0)=\left[\begin{array}{lllll}
0 & 0 & 0.4 & 0 & 0
\end{array}\right], \dot{\mathbf{q}}(0)=\left[\begin{array}{lllll}
0 & 0 & 0 & 0 & 0
\end{array}\right], \\
& r_{1}=r_{2}=r_{3}=1, r_{4}=r_{5}=0.38 \text {, } \\
& K_{11}=K_{12}=K_{13}=2, K_{21}=K_{22}=K_{23}=0.1 \text {, } \\
& \Gamma_{a i}=\Gamma_{u i}=0.2 \\
& b=1 \text {. }
\end{aligned}
$$

It is important to be noted that in practice motions of the bridge, trolley and hoist in a 3D overhead crane are simultaneously controlled, not in order, which were also simulated in these synthetic exercises. Moreover, the control force signals, which were adaptively computed under consideration of influence of the actuator saturation, are illustrated in Fig. 3.

We first examine the simulation results in terms of motions of the bridge, trolley and hoist as the actuated outputs of the system, which are demonstrated in Fig. 4. It was expected that the bridge was required to move from its initial position at $-1 \mathrm{~m}$ to the first desired position at $0.6 \mathrm{~m}$. After $20 \mathrm{~s}$, the bridge was then expected to be returned to the destination at $-0.4 \mathrm{~m}$ before it was intentionally driven to 0 $\mathrm{m}$ at $40 \mathrm{~s}$. As can be seen in Fig. 4a, in the both forward and backward motions, the bridge could steadily converge to the desired positions in less than $5 \mathrm{~s}$ time. Similarly, as illustrated in Fig. 4b, the trolley was expected to move to the left with a distance of $1.2 \mathrm{~m}$ from its starting position at $-0.5 \mathrm{~m}$ and then back to the right with a distance of $1 \mathrm{~m}$ after $20 \mathrm{~s}$ time before it was moved $0.3 \mathrm{~m}$ to a lo- 


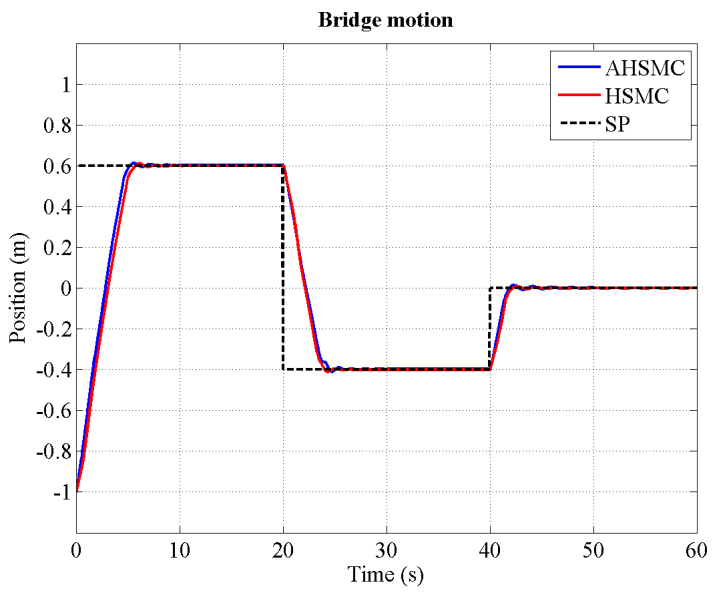

(a)

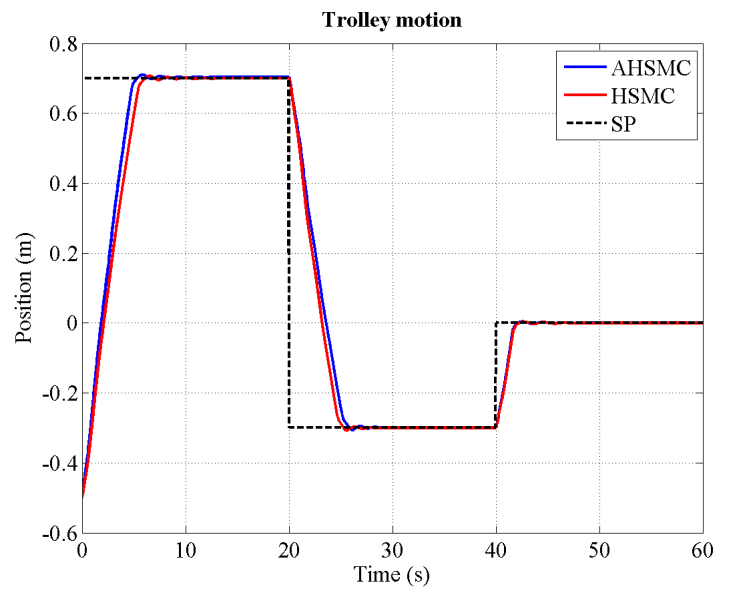

(b)

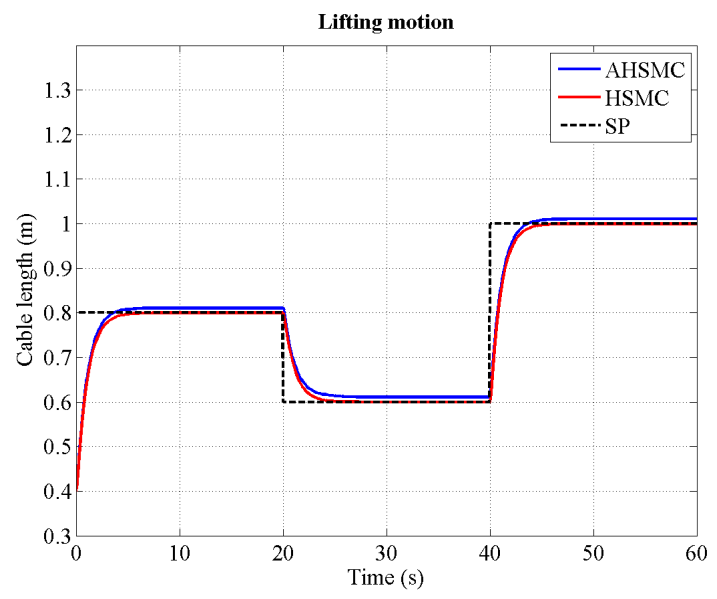

(c)

Figure 4: Simulation actuated output results.

cation of $0 \mathrm{~m}$ at $40 \mathrm{~s}$. It is noticeable that the converging time of the trolley in each motion was also under $5 \mathrm{~s}$. Although the overshoots in both bridge and trolley motions are trivial, the results obtained are highly promising. In contrast, these is no overshoot in the hoisting motions as demonstrated in Fig. 4c. Moreover, though the cargo was required to be first uplifted of $0.4 \mathrm{~m}$, lifted down of $0.2 \mathrm{~m}$ and lifted up of $0.4 \mathrm{~m}$ again at $0 \mathrm{~s}, 20 \mathrm{~s}$ and $40 \mathrm{~s}$, respectively, the responding time of both the lifting and unlifting movements obtained by the proposed algorithm was about $3 \mathrm{~s}$. It can be clearly seen that the actuated outputs in the $3 \mathrm{D}$ crane system obtained by both the HSMC and AHSMC approaches were well tracked the desired positions. Particularly, performance of the AHSMC scheme was highly approximate to that of the HSMC law though some of its parameters are adaptively estimated online. It is noted that performance of the HSMC is the best since its parameters are assumed to be certain while the system parameters in the AHSMC scheme are approximated. Moreover, it can be seen that there is a trivial steady-state error in the hoisting motions shown in Fig. 4c. This negligible error is due to approximation of the unknown and uncertain parameters of the crane system.

More importantly, we investigated the sway angles of the payload, which are illustrated in Fig. 5. It can be clearly seen that those non-actuators responded when the system moved the bridge and trolley to the new destinations and the cargo was lifted up or down. It is noted that all the bridge, trolley and hoist were simultaneously controlled at $0 \mathrm{~s}, 20 \mathrm{~s}$ and $40 \mathrm{~s}$, respectively, which might make the crane not easy to be controlled as compared with the case where the actuators are sequentially controlled. As shown in Fig. $5 \mathrm{a}$, the angle between the projection of the hoist cable on the $\mathrm{X}-\mathrm{Z}$ plane and $\mathrm{Z}$ axis swung at most $0.15 \mathrm{rad}$, while its counterpart, the angle between the hoist cable and its projection on the X-Z plane as demonstrated in Fig. 5b, swung at most $0.1 \mathrm{rad}$, when the system started moving. More particularly, the cargo swings as a result of the AHSMC law are highly comparable with those obtained by the HSMC scheme though the AHSMC strategy does not require some uncertain controlling parameters to be known. 


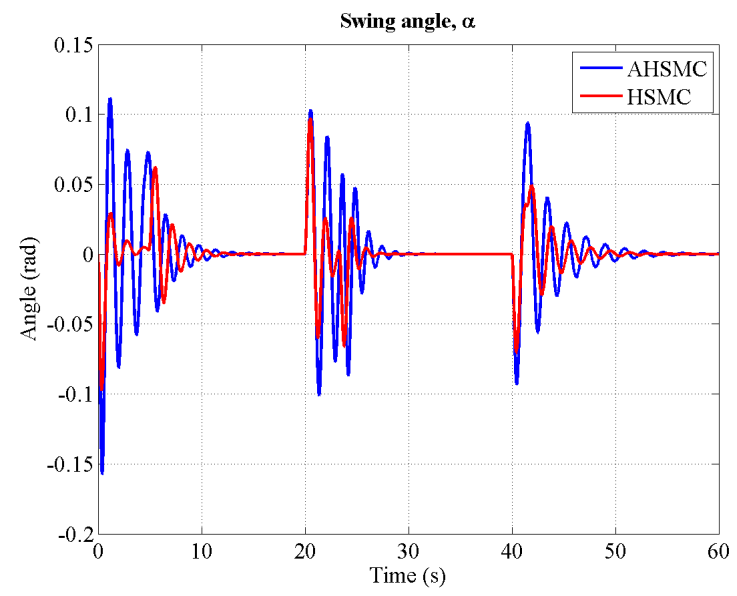

(a)

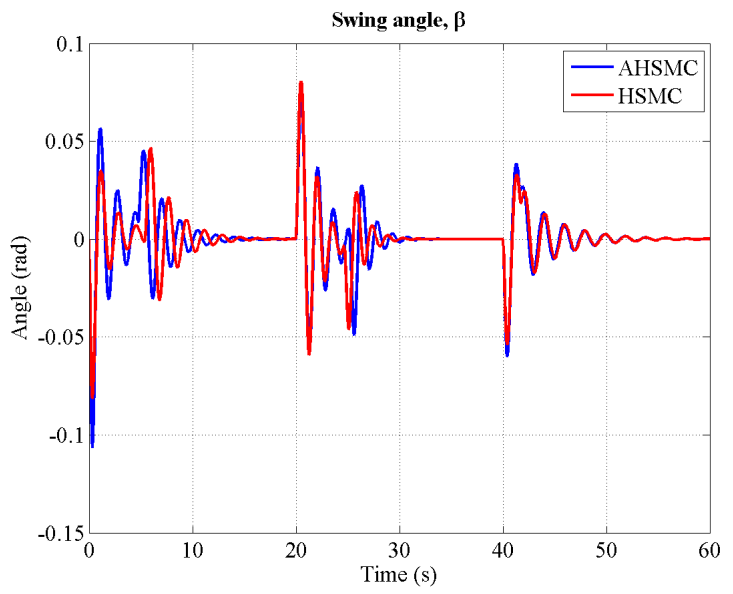

(b)

Figure 5: Simulation un-actuated output results.

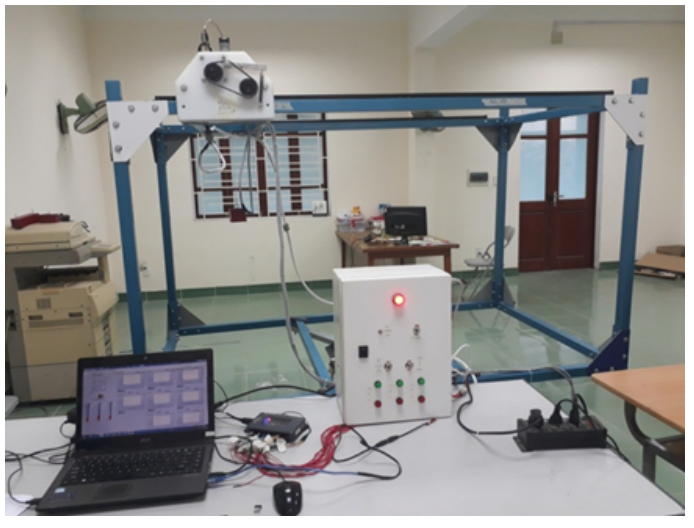

Figure 6: A real-time 3D overhead crane system.

\section{$5.2 \quad$ Experiments}

To experimentally evaluate efficacy of the proposed approach, we implemented the algorithm in a real-life 3D overhead crane system in a laboratory, as shown in Fig. 6. Three actuators in the crane include two three-phase asynchronous motors and one direct current (DC) geared motor. While the alternative current $(\mathrm{AC})$ motors are employed to drive the bridge and trolley, the DC motor is utilized to hoist the payload. Electrically, the AC motors are driven by two Mitsubishi inverters FREQROL-S500, and the DC motor is driven by the $\mathrm{H}$-bridge circuit MB02A manufactured by HBQ Technology, using the pulse width modulation method. Moreover, five encoders with 200-pulse-perround resolution are employed to measure displacements of the bridge, trolley, cable length and swing angles.

Regarding the central control unit, we implemented our algorithm on the NI MyRIO 1900 microcontroller, where the sampling period was set to $10 \mathrm{~ms}$. More particularly, we also designed a human-machine interface by the use of LabView 2014, which enables end-users to set desired positions for the crane, visually monitor the system dynamics and log data for other report and analysis purposes.

By employing the proposed algorithm, the motions of the bridge, trolley and hoist of the experimented crane are illustrated in Fig. 8, while its swing angles of are plotted in Fig. 9. It is noted that the control force signals were adaptively computed by the microcontroller, as shown in Fig. 7. The responses of the experimental crane by the use of our proposed AHSMC scheme are highly similar to those of the simulated system presented in Section 5.1. In other words, converging time of the actuation motions in the simulation is about $5 \mathrm{~s}$ while that in the experiments is about 6 s. Moreover, though the experimented $3 \mathrm{D}$ crane was under realistic disturbances and uncertainties, the overshoots of its motions in transient states as demonstrated in Fig. 8 are trivial. Fig. 9 also shows that the sway angles of the crane were effectively suppressed by the proposed approach though many of its complicated uncertainties are unknown.

Another issue that has been observed in the experimental example as compared to the simulations is the nonzero steady-state errors as shown in both Figures 8 and 9. Though these errors are negligible, the non-zero convergence appearing in reality is partly caused by the realistic nonlinearities and external disturbances of the crane system. On the other hand, since the proposed algorithm was implemented on the microcontroller, the sampling rate also leads to the non-zero convergence of the steady-state errors. Based on the microcontroller's oscillator and the sensors' resolutions, a sampling period of $10 \mathrm{~ms}$ was set to guarantee the microcontroller to be able to run the algorithm in a real time given its computing ability and memory capacity. Though the use of the microcontroller's capability was maximized, the non-zero steady state errors generated by the sampling in the microcontroller were not avoidable in the realistic experiments.

\section{Conclusions}

The paper has proposed an adaptive control scheme for the $3 \mathrm{D}$ uncertain overhead crane based on the hierarchi- 


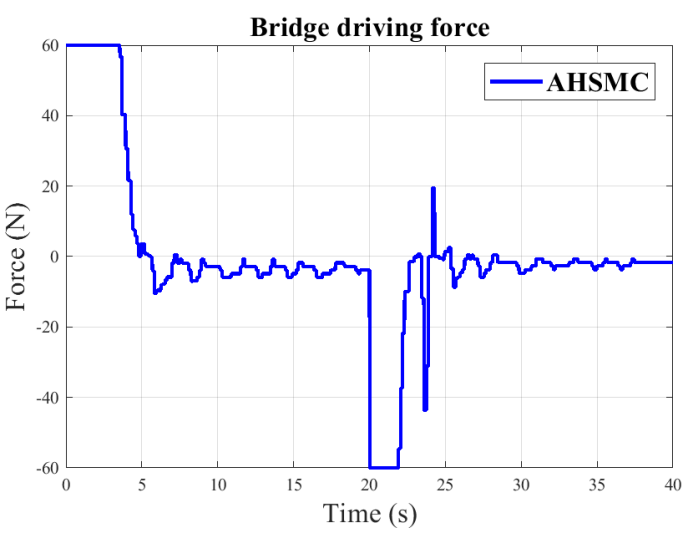

(a)

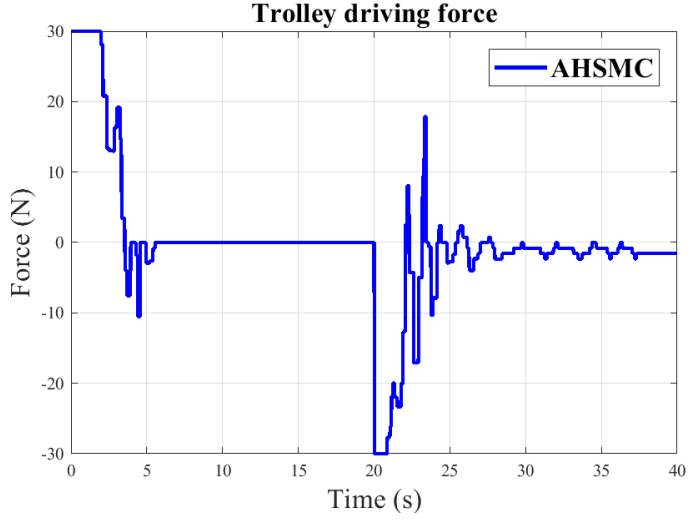

(b)

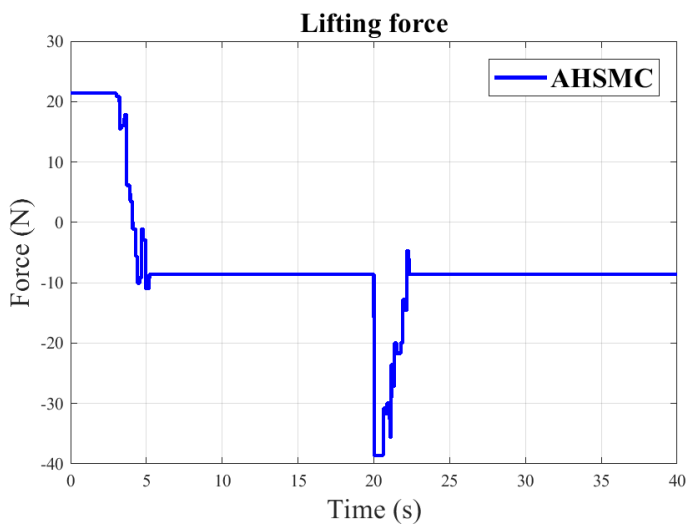

(c)

Figure 7: Experimental control force signals.

cal sliding mode control structure. The controller of the AHSMC law is first designed by the use of the first-order sliding surfaces of both the actuated and un-actuated subsystems. Parameters of the controller are then adaptively estimated by employing the RBFN, which allows the crane to effectively deal with its unknown and non-linear uncertainties and disturbances. The proposed approach was evaluated by both the synthetic and laboratory experiments, where the results obtained by the proposed algorithm AHSMC are highly promising.

\section{References}

[1] H. Butler, G. Honderd, and J. Van Amerongen. Model reference adaptive control of a gantry crane scale model. IEEE Control Systems, pages 57-62, 1991.

[2] Y. Z. Qian, Y. C. Fang, and T. Yang. An energy-based nonlinear coupling control for offshore ship-mounted cranes. International Journal of Automation and Computing, 15(5):570-581, 2018.

[3] Y. Jisup, S. Nation, W. Singhose, and J.E. Vaughan. Control of crane payloads that bounce during hoisting. IEEE Transactions on Control Systems Technology, 22:1233-1238, 2014.
[4] L. Ramli, Z. Mohamed, A. M. Abdullahi, H. I. Jaafar, and I. M. Lazim. Control strategies for crane systems: A comprehensive review. Mechanical Systems and Signal Processing, 95:1-23, 2017.

[5] F. Panuncio, W. Yu, and X. Li. Stable neural pid antiswing control for an overhead crane. In Proc. IEEE International Symposium on Intelligent Control, pages 53-58, Hyderabad, India, August 2013.

[6] H. Lee. Motion planning for three-dimensional overhead cranes with high-speed load hoisting. International Journal of Control, 78(12):37-41, 2007.

[7] N. Sun, Y. Fang, Y. Zhang, and B. Ma. A novel kinematic coupling-based trajectory planning method for overhead cranes. IEEE/ASME Transactions on Mechatronics, 17(1):166-173, 2012.

[8] H. Saeidi, M. Naraghi, and A.A. Raie. A neural network self tuner based on input shapers behavior for anti sway system of gantry cranes. Journal of Vibration and Control, 19:1936-1949, 2013.

[9] H. M. Omar. Control of gantry and tower cranes. PhD thesis, Virginia Polytechnic Institute and State University, 2003. 


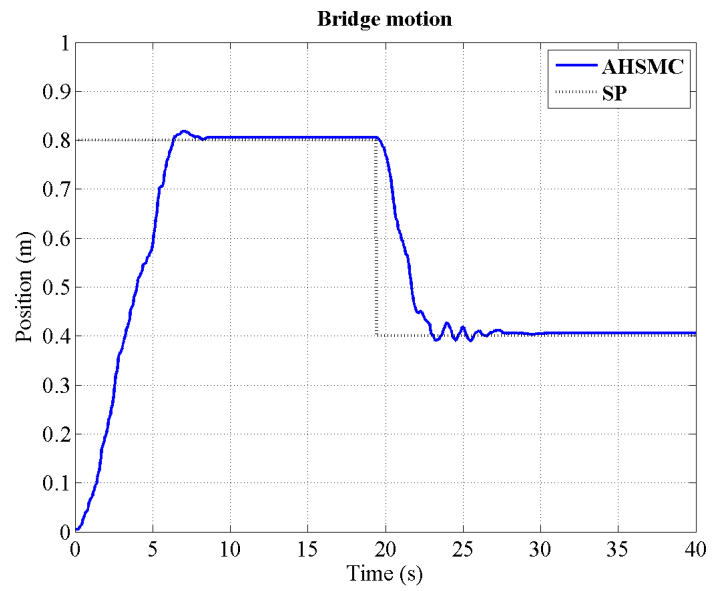

(a)

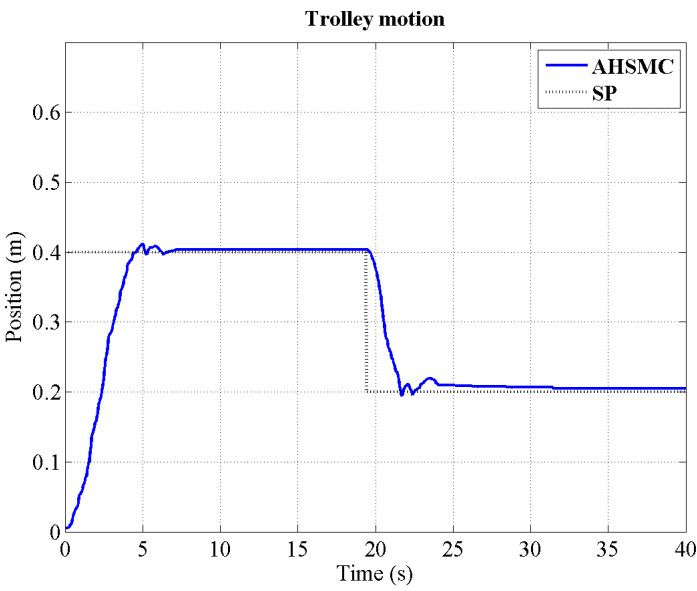

(b)

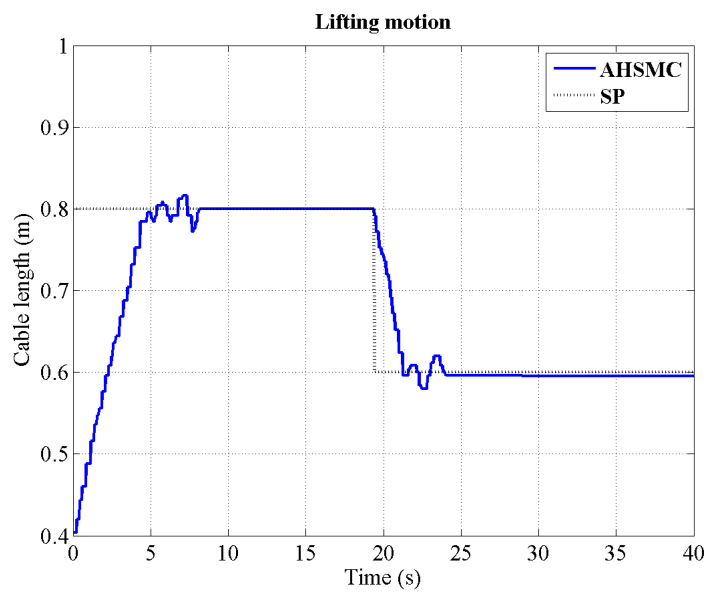

(c)

Figure 8: Experimental actuated output results.

[10] H. M. Omar and A. H. Nayfeh. Anti-swing control of gantry and tower cranes using fuzzy and time delayed feedback with friction compensation. Shock and Vibration, 12:73-89, 2005.

[11] Y. Fang, W. E. Dixon, D. M. Dawson, and E. Zergeroglu. Nonlinear coupling control laws for an overhead crane system. IEEE/ASME Transactions on Mechatronics, 8(3):418-423, 2003.

[12] J. Yu, F. L. Lewis, and T. Huang. Nonlinear feedback control of a gantry crane. In Proc. American Control Conference, pages 4310-4315, Seattle, WA, USA, June 1995.

[13] H. Park, D. Chwa, and K. Hong. A feedback linearization control of container cranes: Varying rope length. International Journal of Control, Automation and Systems, 5(4):379-387, 2007.

[14] T. A. Le, S. Lee, V. Dang, S. Moon, and B. Kim. Partial feedback linearization control of a threedimensional overhead crane. International Journal of Control, Automation and Systems, 11(4):718-727, 2013.

[15] T. A. Le, G. Kim, M. Y. Kim, and S. Lee. Partial feedback linearization control of overhead cranes with varying cable lengths. International Journal of Precision Engineering and Manufacturing, 13(4):501-507, 2012.

[16] S. Cho and H. Lee. A fuzzy-logic antiswing controller for three-dimensional overhead cranes. ISA Transactions, 41(2):235-243, 2002.

[17] M. Mahfouf, C. H. Kee, M. F. Abbod, and D. A. Linkens. Fuzzy logic-based anti-sway control design for overhead cranes. Neural Computing and Applications, 9(1):38-43, 2000.

[18] L. Wang, H. Zhang, and Z. Kong. Anti-swing control of overhead crane based on double fuzzy controllers. In Proc. 27th Chinese Control and Decision Conference, pages 981-986, Qingdao, China, 2015. 


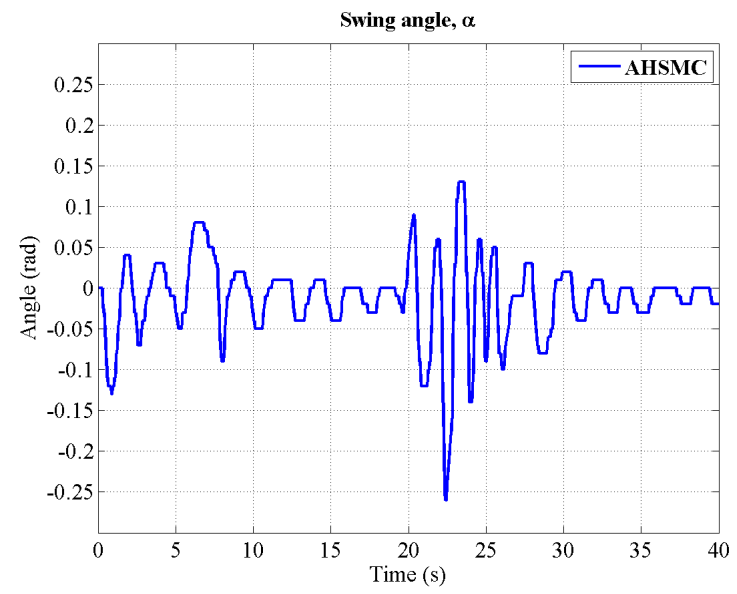

(a)

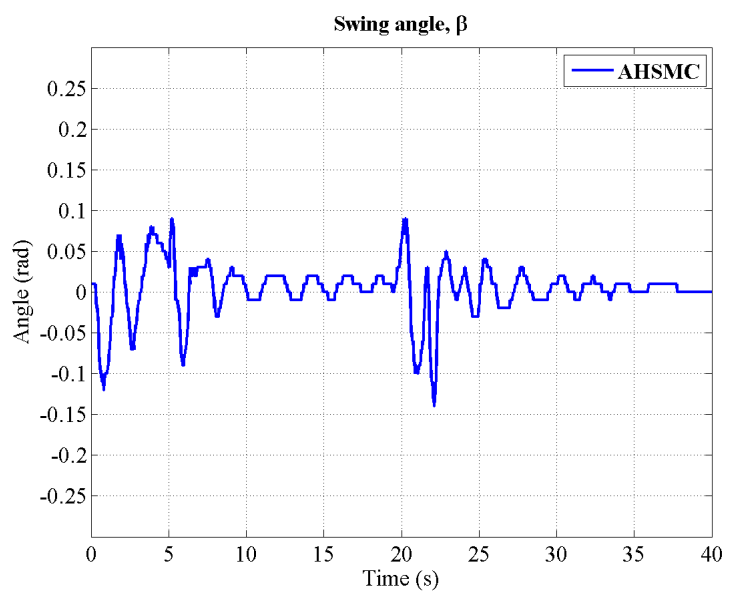

(b)

Figure 9: Experimental un-actuated output results.

[19] K. Shyu, C. Jen, and L. Shang. Design of sliding-mode controller for anti-swing control of overhead cranes. In Proc. 31st Annual Conference of IEEE Industrial Electronics Society, pages 147-152, Raleigh, NC, USA, November 2005.

[20] D. Qian, J. Yi, and D. Zhao. Control of overhead crane systems by combining sliding mode with fuzzy regulator. IFAC Proceedings Volumes, 44(1):9320-9325, 2011.

[21] T. A. Le, J. Kim, S. Lee, T. Lim, and N. C. Luong. Second-order sliding mode control of a $3 \mathrm{D}$ overhead crane with uncertain system parameters. International Journal of Precision Engineering and Manufacturing, 15(5):811-819, 2014.

[22] Q. H. Ngo and K. Hong. Sliding-mode antisway control of an offshore container crane. IEEE/ASME Transactions on Mechatronics, 17(2):201-209, 2012.

[23] G. Bartolini, A. Pisano, and E. Usai. Second-order sliding-mode control of container cranes. Automatica, 38(10):1783-1790, 2002.

[24] S. Mahjoub, F. Mnif, and N. Derbel. Second-order sliding mode approaches for the control of a class of underactuated systems. International Journal of Automation and Computing, 12(2):134-141, 2015.

[25] W. Xu, X. Zheng, Y. Liu, M. Zhang, and Y. Luo. Adaptive dynamic sliding mode control for overhead cranes. In Proc. 34th Chinese Control Conference, pages 3287-3292, Hangzhou, China, July 2015.

[26] W. Wang, J. Yi, D. Zhao, and D. Liu. Design of a stable sliding-mode controller for a class of secondorder underactuated systems. IEE Proceedings - Control Theory and Applications, 151(6):243-250, 2004.

[27] D. Qian, J. Yi, and D. Zhao. Hierarchical sliding mode control for a class of SIMO underactuated system. Control and Cybernetics, 37(1):160-175, 2008.
[28] D. Qian and J. Yi. Hierarchical Sliding Mode Control for Under-actuated Cranes: Design, Analysis and Simulation. Springer, 2016.

[29] H. X. Le, T. V. Nguyen, A. V. Le, N. T. T. Vu, and M. X. Phan. Adaptive backstepping hierarchical sliding mode control for uncertain 3d overhead crane systems. In Proc. IEEE International Conference on System Science and Engineering, pages 438-443, Ho Chi Minh City, Vietnam, September 2017.

[30] W. Wang, X. D. Liu, and J. Q. Yi. Structure design of two types of sliding-mode controllers for a class of under-actuated mechanical systems. IET Control Theory and Applications, 1(1):163-172, 2007.

[31] D. Qian, X. Liu, and J. Yi. Adaptive control based on hierarchical sliding mode for under-actuated systems. In Proc. International Conference on Mechatronics and Automation, pages 1050-1055, Chengdu, China, August 2012.

[32] J. H. Yang and K. S. Yang. Adaptive coupling control for overhead crane systems. IET Control Theory and Applications, 17(2-3):143-152, 2007.

[33] T. A. Le, S. Lee, N. C. Luong, and D. H. Kim. Model reference adaptive sliding mode control for three dimensional overhead cranes. International Journal of Precision Engineering and Manufacturing, 14(8):13291338, 2013.

[34] M. Park, D. Chwa, and S. Hong. Antisway tracking control of overhead cranes with system uncertainty and actuator nonlinearity using an adaptive fuzzy slidingmode control. IEEE Transactions on Industrial Electronics, 55(11):3972-3984, 2008.

[35] M. Park, D. Chwa, and M. Eom. Adaptive slidingmode antisway control of uncertain overhead cranes with high-speed hoisting motion. IEEE Transactions on Fuzzy Systems, 22(5):1262-1271, 2014. 
[36] L. Hung and H. Chung. Decoupled control using neural network-based sliding-mode controller for nonlinear systems. Expert Systems with Applications, 32(4):1168-1182, 2007.

[37] Geng Ji. Adaptive neural network dynamic surface control for perturbed nonlinear time-delay systems. International Journal of Automation and Computing, 9(2):135-141, 2012.

[38] L. Hung and H. Chung. Decoupled sliding-mode with fuzzy-neural network controller for nonlinear systems. International Journal of Approximate Reasoning, 46(1):74-97, 2007.

[39] C. Tsai, H. L. Wu, and K. Chuang. Intelligent slidingmode motion control using fuzzy wavelet networks for automatic $3 \mathrm{~d}$ overhead cranes. In Proc. SICE Annual Conference, pages 1256-1261, Akita, Japan, Agust 2012.

[40] Y. Tao, J. Zheng, and Y. Lin. A sliding mode controlbased on a rbf neural network for deburring industry robotic systems. International Journal of Advanced Robotic Systems, 2016.

[41] S. Mahjoub, F. Mnif, and N. Derbel. Radial-basisfunctions neural network sliding mode control for underactuated manipulators. In Proc. 10th MultiConferences on Systems, Signals \& Devices, Hammamet, Tunisia, March 2013.

[42] H. C. Lu, C. H. Tsai, and M. H. Chang. Radial basis function neural network with sliding mode control for robotic manipulators. In Proc. IEEE International Conference on Systems, Man and Cybernetics, Istanbul, Turkey, October 2010.

[43] S. Mahjoub, F. Mnif, N. Derbel, and M. Hamerlain. Radial-basis-functions neural network sliding mode control for underactuated mechanical systems. International Journal of Dynamics and Control, 2:533$541,2014$.

[44] Z. Chao, Z. Aimin, Z. Hang, B. Yunfei, G. Chujia, and G. Yingsan. Rbf neural networks sliding mode controller design for static var compensator. In Proc. 34th Chinese Control Conference, Hangzhou, China, July 2015.

[45] H. Lee. Modeling and control of a three-dimensional overhead. Journal of Dynamic Systems, Measurement, and Control, 120(4):471-476, 1998.

[46] R. Selmic and F. L. Lewis. Deadzone compensation in motion control systems using neural networks. IEEE Transactions on Automatic Control, 45(4):602613,2000

[47] J. Zhou, C. Wen, and T. Li. Adaptive output feedback control of uncertain nonlinear systems with hysteresis nonlinearity. IEEE Transactions on Automatic Control, 57(10):2627-2633, 2012.

[48] J. Park and I. W. Sandberg. Structure design of two types of sliding-mode controllers for a class of underactuated mechanical systems. Neural Computation, 3(2):246-257, 1991.

[49] M. T. Hagan, H. B. Demuth, M. H. Beale, and O. D. Jess. Neural Network Design. Martin Hagan, USA, 2014.

[50] Wenzhi Gao and Rastko R Selmic. Neural network control of a class of nonlinear systems with actuator saturation. IEEE Transactions on Neural Networks, 17(1):147-156, 2006. 